\title{
Prof. Dr. Moussa B.H. Youdim: an appreciation on the occasion of his 80th birthday
}

\author{
Peter Riederer ${ }^{1}$ \\ Published online: 3 February 2020 \\ (c) Springer-Verlag GmbH Austria, part of Springer Nature 2020
}

Moussa Youdim's $(* 1940)$ international career had already started by the late 1940s when he left Teheran to pursue his education in London, finishing at Borough Polytechnic with an "A" level in botany, chemistry, physics and zoology. He continued his studies from 1960 to 1966 at McGill University in Montreal, Canada, where he earned a PhD in biochemistry. Here- under the mentorship of Theodore Sourkes-he began working on the isolation and characterization of monoamine oxidase (MAO), including first studies on MAO enzymes.

He then went back to London (1966-1972) as a Senior Research Associate at the University of London and as a postdoctoral fellow at the Bernhard Baron Memorial Labs, where he got in touch with its head, Merton Sandler, who became a mentor and friend of his and mine for several decades. After stays in Paris and Oxford, he became Chair and Professor of Pharmacology at the Technion in Haifa in 1977, where he is still conducting research in retirement today.

Moussa Youdim is one of the pioneers of MAO research, starting from its enzyme's neurochemistry, moving to its neuropharmacological aspects, and translating this knowledge into medicinal products. Deciphering the fundamental roles of MAO-A and -B and, in particular, the metabolism of dopamine, he contributed heavily to the oxidative stress hypothesis as well as to our knowledge of the iron-induced toxicity of nerve cells in neurodegenerative disorders, especially Parkinson's disease (PD). These studies led to the development of the anti-Parkinson's drugs selegiline and rasagiline as well as the multimodal anti-Alzheimer's disease (AD) drug ladostigil. Other developments include preclinical studies on iron chelators that cross the blood-brain barrier, and are inhibitors of MAO and possibly of cholinesterase.

Peter Riederer

peter.riederer@uni-wuerzburg.de

1 University Hospital Wuerzburg, Clinic and Policlinic for Psychiatry, Psychosomatics and Psychotherapy, Margarete Hoeppel-Platz 1, 97080 Wuerzburg, Germany
Moussa Youdim is also one of the few academics who employs the entire armamentarium of biochemistry, neurochemistry, neuropharmacology, molecular biology, transcriptomics, proteomic profiling, and behavioural findings. In this way, he and his team made fundamental contributions and developments in the management of neurodegenerative disorders, such as the development of rasagiline, TVP 1022 capillary endothelial factor VIII clotting factor, and neuroprotective/neurorescue agents as anti-PD and anti-AD agents (TVP 136, TVP 137, anti-cholinesterase inhibitors ladostigil and TVP 3279 for the treatment of Alzheimer's disease, and the iron chelators VK-28 and M-30).

While drug development based on findings from human post-mortem brain studies and experimental studies in animal models of PD and AD is a major focus of his scientific work, more recent interests focus on the discovery of biomarkers for both, the early detection of PD, and markers for disease progression. This is absolutely crucial, since such biomarkers would allow PD to be treated even before major symptomology emerges and at a time when neurons could still be protected from degenerative processes. On the other hand, biomarkers for characterizing the progression of PD would help clinicians in optimizing drug treatment and obtaining information about future developments of $\mathrm{PD}$ patients' prognosis and treatment potentials.

Moussa Youdim has been the recipient of numerous prizes, awards and honors, such as the Anna-Monika Prize for the neurochemistry of depression (Switzerland), 1972; Honorary Membership in the Austrian Neurological Society, 1986; the Claudius Galenius Gold Medal Prize for the Parkinson's Disease L-Deprenyl Drug of the Year (Berlin, Germany), 1991; the AGNP-Prize (Nurnberg, Germany), 1991; a Honorary Membership in the International College of Neuropsychopharmacology (CINP), 1994; Doctor Honoris Causa, Semmelweis University, Budapest, Hungary, 1997; the World Federation of Neurology Award, Amsterdam, Netherland, 2007; Distinguished Chair Professor, Hong Kong University; as well as at the University Qingdao, 
China, 2008 and at the Yonsei University of Seoul, South Korea, 2008.

More than 600 publications in international scientific journals have demonstrated his innovational potential for enlarging the armamentarium to combat such disastrous diseases as Parkinson's and Alzheimer's. I am happy to have had the opportunity to cooperate scientifically with him for over 45 years now, with many fruitful discussions, experimental interactions, and drug developments for the benefit of patients suffering from neurodegenerative disorders.

My personal friendship with Moussa started at the International Congress for Biochemistry that was held in Stockholm, Sweden, in July 1973. At this congress, we presented posters related to the metabolism of biogenic amines. Our posters were located in the same room and contact between the two of us started easily and quickly. My poster on "Alterations of precursors and metabolites of biogenic amines in Parkinson- and depression syndromes" was of interest to Moussa, since his poster took up monoamine oxidase (MAO) in depression. Indeed, we had a long discussion on these topics and sat down on the floor in front of Moussa's poster for the next hour to broaden our view of the role of monoamine oxidase.

In 1973, Madopar (levodopa plus the peripherally acting decarboxylase inhibitor benserazide) came on the market and Walther Birkmayer recognized off-phases in PD patients. Our neurochemical work was related to this clinical observation, and my conclusion was to try a MAO inhibitor. At my research stay at the Bernhard Baron Memorial Research Institute in London in November 1973, I discussed this idea with the head of the laboratories, Merton Sandler, and on his advice with Moussa as well, who was at that time working in Oxford with Graham-Smith on the behavior of irondepleted rats. This meeting was decisive, as it ultimately resulted in the use of L-deprenyl (selegiline) from October 1974 onwards, with the first publication following in 1975.

We then worked together on iron-induced oxidative stress and neuromelanin, and cooperated especially in developing selegiline. Our cooperation resulted in many contributions at congresses and symposia, we published several books and many scientific papers together, and received some honors for our work, too.

Despite our different personalities, or maybe because of them, we were able to develop a mutual enthusiasm as the driving force behind performing neuroscience for the benefit of patients with neuropsychiatric disorders.

We had and continue to enjoy a great friendship, and I will not forget the numerous private meetings in Haifa and at places around the globe.

Publisher's Note Springer Nature remains neutral with regard to jurisdictional claims in published maps and institutional affiliations. 